\title{
EDITORIAL
}

\section{How I treat septic shock}

\author{
Jean-Louis Vincent ${ }^{*}$ (1)
}

C 2018 Springer-Verlag GmbH Germany, part of Springer Nature and ESICM

\section{Introduction}

Septic shock is the worst form of sepsis, associated with acute circulatory failure and hyperlactatemia $[1,2]$. Septic shock is an emergency, with every aspect of management a matter not of hours but of minutes, so I make sure my team has enough people to complete all the necessary interventions efficiently and effectively, under my leadership. My patient management is based on the three major components shown in Fig. 1; importantly, infection and hemodynamic management must be performed simultaneously.

\section{Infection management}

Antibiotics are effective, so it makes sense to administer them as quickly as possible. I use the antibiotics most likely to cover all potential organisms but this does not mean that I give every patient broad-spectrum antibiotics. For example, in our hospital, patients with community-acquired peritonitis can be effectively treated initially with amoxicillin/clavulanic acid. Nevertheless, combination therapy is currently advised in septic shock; I usually add amikacin (I do not trust quinolones very much in critically ill patients), sometimes only for a single dose. Of course, if there is any possibility of staphylococcal infection, even in our unit where methicillin-resistant Staphylococcus aureus (MRSA) is no longer common, I add vancomycin. Every member of the team knows that all possible samples for culture must be rapidly obtained before antibiotics are given.

When the source of infection is not evident, I reassess the "big five" likely culprits-lungs, abdomen, urinary tract, skin, and catheters-and encourage the nurses to be involved in the search, particularly for skin and catheter-related infection, as they usually look at these better than we do! If a procedure needs to be done, e.g., surgical

*Correspondence: jlvincent@intensive.org

Department of Intensive Care, Erasme University Hospital, Université libre de Bruxelles, Route De Lennik 808, 1070 Brussels, Belgium drainage or catheter removal, I make sure it is done as soon as possible, personally engaging with operating room or interventional radiology staff when necessary.

\section{Hemodynamic management}

Hemodynamic management is conducted in four phases, summarized by the letters SOSD—salvage, optimization, stabilization, and de-escalation [1]; importantly, each phase has a different duration and durations vary in different patients.

\section{$S$ for salvage}

In this initial resuscitation phase, my goal is to urgently restore some degree of organ perfusion. Fluids and vasopressor agents are given quickly before much monitoring equipment has been set up. I do not follow any specific protocol for fluid administration, but usually give a first liter (adapted roughly according to the patient's body weight) of intravenous fluid at a fast rate. I then give $1 \mathrm{l} / \mathrm{h}$ for a brief period, during initial monitoring with echocardiography. If the condition is severe, I introduce a central venous catheter (or rather, invite a junior doctor to do so!). These two interventions can be achieved in less than 30 min in all patients.

I usually use a crystalloid as my initial fluid and prefer balanced solutions (Ringer's lactate or PlasmaLyte). If I use normal saline (in patients without severe acidosis), I check chloride levels regularly (at least after each liter of saline solution) [3] to ensure hyperchloremia does not develop. In patients with hypoalbuminemia (typically albumin levels $<2.2 \mathrm{~g} / \mathrm{dl}$, although there is no strict cut-off) who are already edematous (e.g., patients with decompensated cirrhosis), I may use albumin.

I use norepinephrine as the vasopressor of choice and start it at virtually the same time as fluids. I do not believe we need to wait for the response to fluids to be evaluated before we start vasopressor therapy. I individualize the doses of norepinephrine needed to achieve an adequate mean arterial pressure (not $65 \mathrm{mmHg}$ for

\section{实




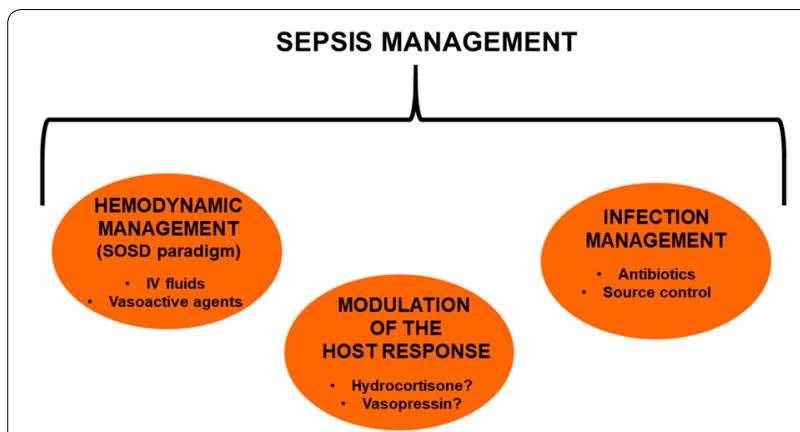

Fig. 1 The three components of septic shock management. SOSD salvage, optimization, stabilization, and de-escalation

everyone!). Dopamine should no longer be used. I also avoid epinephrine because I am concerned that it is more arrhythmogenic, may reduce splanchnic blood flow, and may alter cellular metabolism.

\section{O for optimization}

Fluid administration must be optimized to ensure adequate tissue perfusion by increasing cardiac output while limiting increase in filling pressures and development of edema. In all patients, I use repeated fluid challenges to guide ongoing fluid administration. For a fluid challenge, I give a small amount of fluid (100-200 ml) over $10 \mathrm{~min}$ and watch the dynamic effect on cardiac output and central venous pressure (CVP) [4]. A minimal change in CVP along with an increase in cardiac output suggests that fluid is beneficial and fluids are continued. A large increase in CVP with little change in cardiac output indicates poor fluid tolerance and fluid infusion is immediately stopped. In mechanically ventilated patients who have no spontaneous breathing, I assess the pulse pressure variation (usually visually) or stroke volume variation (using pulse contour analysis) but this situation is rare, because we try to minimize sedative use in our unit. In patients with complex hemodynamic conditions, I still use a pulmonary artery catheter in addition to repeated echocardiographic evaluations, according to the current guidelines [5].

If signs of altered tissue perfusion persist but fluids are no longer tolerated (i.e., there is an increase in cardiac filling without an increase in cardiac output), I add a small dose of dobutamine $(3-5 \mu \mathrm{g} / \mathrm{kg} / \mathrm{min}$ is usually sufficient) [6]. Severe peripheral vasoconstriction is an incentive to give it. Despite the negative studies on early goal-directed therapy [7], I check the central venous oxygen saturation $\left(\mathrm{ScrO}_{2}\right)$, because a low value $(<70 \%)$ can help support the decision to give some dobutamine or a blood transfusion if the hemoglobin concentration is decreased [8]. I measure blood lactate levels every hour during shock [9] to check they are decreasing. If lactate levels stagnate or even increase, I reconsider my strategy and may contact the surgeon or the radiologist to reassess source control.

\section{S for stabilization}

This period is best summarized simply by the four letters, STOP. The patient is improving, so we stop fluid resuscitation and move to maintenance fluids. Vasopressor doses are stable or can already start to be decreased.

\section{D for de-escalation}

The patient is now clearly improving, so we wean from vasopressor agents and limit fluid intake. If the patient does not eliminate any excess fluid, I give diuretics [or add ultrafiltration as part of renal replacement therapy (RRT)], but this is a rare event. Some people call this phase "de-resuscitation", but this term is inappropriate, because it suggests a backward step to the time prior to resuscitation.

\section{Modulation of host response and other aspects of patient management}

Our ability to modulate the host response is still limited. In patients with severe septic shock, I believe there is now good evidence that administration of moderate doses of hydrocortisone (200 mg/day in four doses) improves outcomes [10]. I do not believe that fludrocortisone is necessary. I consider vasopressin as a form of compensation for relative vasopressin deficiency and prescribe it at limited doses $(0.03 \mathrm{U} / \mathrm{min})$ in the rare cases when vascular tone is extremely reduced, i.e., when hypotension persists in the presence of a high cardiac output. Some people overuse vasopressin, forgetting it can be very harmful if cardiac output is not elevated.

I add vitamins only in cases of malnutrition and I do not give selenium. I avoid enteral nutrition during the shock phase, because there is a risk of gut ischemia. Unless contraindicated, I start nutritional support during the stabilization phase.

\section{Conclusion}

Patients with septic shock require rapid, effective, and complete management by a trained group of individuals. Every minute counts to limit organ dysfunction, and good treatment can make a clear difference in complication rates and survival. Many factors, including bacterial pathogenicity, the time course, and various host features such as immune status and comorbidities, can influence outcomes. Hence, I apply individualized treatment, guided by appropriate monitoring systems. Because of the complex nature of these patients and the need for 
multiple, diverse, and rapid management strategies, a team approach is required $24 \mathrm{~h}$ a day, 7 days a week.

\section{Compliance with ethical standards}

\section{Conflicts of interest}

The author has no conflicts of interest to declare related to this manuscript.

\section{Ethical approval}

This article does not contain any studies with human participants or animals performed by the author.

\section{Received: 31 July 2018 Accepted: 1 October 2018}

Published online: 12 October 2018

\section{References}

1. Vincent JL, De Backer D (2014) Circulatory shock. N Engl J Med 370:583

2. Singer M, Deutschman CS, Seymour CW, Shankar-Hari M, Annane D, Bauer M, Bellomo R, Bernard GR, Chiche JD et al (2016) The third international consensus definitions for sepsis and septic shock (Sepsis-3). JAMA 315:801-810

3. Vincent JL, De Backer D (2018) We do not appreciate SALT. Am J Respir Crit Care Med 197:1361

4. De Backer D, Vincent JL (2018) Should we measure the central venous pressure to guide fluid management? Ten answers to 10 questions. Crit Care 22:43

5. Cecconi M, De BD, Antonelli M, Beale R, Bakker J, Hofer C, Jaeschke R, Mebazaa A, Pinsky MR et al (2014) Consensus on circulatory shock and hemodynamic monitoring. Task force of the European Society of Intensive Care Medicine. Intensive Care Med 40:1795-1815

6. Vincent JL, Roman A, Kahn RJ (1990) Dobutamine administration in septic shock: addition to a standard protocol. Crit Care Med 18:689-693

7. De Backer D, Vincent JL (2016) Early goal-directed therapy: do we have a definitive answer? Intensive Care Med 42:1048-1050

8. Vincent JL, De Backer D (2018) From early goal-directed therapy to late(r) $\mathrm{ScvO}_{2}$ checks. Chest (in press)

9. Vincent JL, e Silva AQ, Couto L Jr, Taccone FS (2016) The value of blood lactate kinetics in critically ill patients: a systematic review. Crit Care 20:257

10. Annane D, Renault A, Brun-Buisson C, Megarbane B, Quenot JP, Siami S, Cariou A, Forceville X, Schwebel C et al (2018) Hydrocortisone plus fludrocortisone for adults with septic shock. N Engl J Med 378:809-818 